\title{
Estudio de los factores que inciden en la baja producción y comercialización de los cultivos de maíz.
}

Study of factors that affect the low production and marketing of maize crops.

Ramiro Enrique Guamán Chávez. MSc. ${ }^{1}$, Evelyn Eugenia Alcívar Soria, MAE. ${ }^{2}$, López Martha Cecilia, MAE. ${ }^{3} \&$ Leici Edid Quiñónez Cabeza MAE. ${ }^{4}$

DOI: $\underline{\text { https://doi.org/10.33262/visionariodigital.v2i1.36 }}$

\section{Resumen.}

El presente trabajo de investigación relacionado con estudio de los factores que inciden en la baja producción y comercialización de los cultivos de maíz de los agricultores del cantón buena fe, de la provincia de los Ríos, se trata de analizar los factores están influyendo para que existan bajos rendimientos de producción y mala comercialización del cultivo de maíz, para su desarrollo fue necesario aplicar los métodos crítico propositivo con el nivel exploratorio, explicativo y descriptivo; como técnicas se aplicaron los diálogos formales e informales entrevistas, encuestas, arrojando un alto porcentaje productores que no cuenta con asistencia técnica alguna. Esto se debe a que no tienen la capacitación oportuna para mejorar su productividad y competitividad y que factores como crédito oportuno, asistencia técnica, insumos de calidad y comercialización de la gramínea son imprescindibles en la rentabilidad en el cultivo de maíz. Que es necesaria la capacitación sobre comercialización al agricultor de cultivo de maíz del cantón Buena $\mathrm{Fe}$, además aseguran que si participarían de las actividades para la capacitación sobre comercialización de sus productos, de igual forma sus beneficios serían muy importantes, esto mejorarían las ventas.

1 Universidad Técnica Luis Vargas Torres de Esmeraldas Extensión La Concordia. Ecuador Ramiro.guaman@utelvt.edu.ec

2 Universidad Técnica Luis Vargas Torres de Esmeraldas Extensión La Concordia, Ecuador, evelyn_alcivar@hotmail.com

3 Universidad Técnica Luis Vargas Torres de Esmeraldas Extensión La Concordia, Ecuador, cecilia.lopez@utelvtg.edu.ec

4 Universidad técnica Luis Vargas Torres de Esmeraldas extensión La Concordia, Ecuador, leici.quinonez@utelvt.edu.ec 
www.visionariodigital.org

Palabras clave: Producción agrícola, cultivos, comercialización, agricultor, economía agraria.

\begin{abstract}
.
This research work related to the study of the factors that affect the low production and commercialization of corn crops of farmers in the canton of good faith, in the province of Los Ríos, is to analyze the factors that are influencing the existence of low yields of production and bad commercialization of the corn crop, for its development it was necessary to apply the critical - proactive methods with the exploratory, explanatory and descriptive level; As formal and informal dialogues, interviews and surveys were applied, yielding a high percentage of producers that do not have any technical assistance. This is because they do not have the opportune training to improve their productivity and competitiveness and factors such as timely credit, technical assistance, quality inputs and marketing of the grass are essential in the profitability of growing corn. That the training on marketing to the farmer of corn cultivation of the canton Buena Fe is necessary, also assure that if they would participate in the activities for the training on marketing of their products, in the same way their benefits would be very important, this would improve the sales.
\end{abstract}

Keywords: Agricultural production, crops, commercialization, farmer, agrarian economy.

\title{
I. Introducción.
}

En Latinoamérica las fluctuaciones en los precios son un hecho en todas las ramas de la economía, precisamente, esos cambios permanentes en los precios reflejan todos los factores que afectan la oferta y demanda de cualquier producto. Así por ejemplo, una prolongada sequía influye en el rendimiento de la cosecha de maíz, y probablemente provoque un aumento en el precio del poroto, lo que también hará que las industrias deban pagar más por la materia prima que necesitan para moler, esto hará que los consumidores tengan que pagar más el aceite que compran en el supermercado (Philip \& Gary , 2012).

Precisamente, porque nadie puede con certeza predecir lo que pasará mañana con los precios, los Mercados de Futuros, que los hay en todo el mundo, existen para minimizar o acotar el impacto que producen los cambios en los precios de las mercaderías. En los últimos años, los COMMODITIES agrícolas se han vuelto particularmente volátiles, aumentando el riesgo precio que soportan los participantes comerciales del mercado de granos. Esas mismas personas más de una vez se habrán preguntado ¿Cómo puedo obtener algún tipo de protección contra las variaciones de los precios de mi mercadería o la materia prima que debo adquirir? ¿Podré beneficiarme alguna vez de esas variaciones de precios? ( Hada , 2008).

Si es un productor, acopio, industrial o exportador muy probablemente tenga interés en protegerse contra los cambios adversos en los precios. Como así también si es inversor también querrá anticiparse a los cambios que tendrán los precios y poder obtener una 
www.visionariodigital.org

ganancia. Actualmente en Ecuador una parte importante del país la creciente demanda de ésta gramínea ya sea para el consumo directo en la alimentación humana, o para suministrar alimento a otros sectores de la producción, para la industria en general o para su exportación, hace evidente la necesidad de manejar a éste cultivo en forma adecuada para lograr una mayor producción y una eficiente comercialización (Zarifian, 1999)

La producción nacional de ésta gramínea varía debido a diferentes factores. En nuestro país, el rendimiento estimado por hectárea es de 3.7 TM para el nivel tecnificado, encontrándose por debajo de los internacionales comparado con el de los Estados Unidos que es de 7 TM por hectárea. Esto a pesar de que el Ecuador por encontrarse en una ubicación geográfica estratégica en el planeta cuenta con regiones de excepcionales características EDAFO-climáticas que le permiten desarrollar una amplia diversidad de cultivos tanto tradicionales como no tradicionales (CEPAL, 2016).

Entre los factores que limitan el alcance de los niveles de producción para que cumplan con las expectativas del mercado local e internacional, tenemos: la falta de asistencia técnica y transferencia de tecnología, fallas en el sistema de comercialización y la escasez de líneas de crédito sobre todo para los pequeños y medianos productores, que son las que a la final se convierten en barreras que impiden un normal desarrollo dentro de esta actividad, produciéndose de ésta manera un estancamiento en el incremento de los ingresos de los productores maiceros y de divisas para el país (Arce , 2009).

En los últimos años la Baja producción por hectárea de los cultivos de maíz de los Agricultores del Cantón Buena Fe de la Provincia de Los Ríos, ha generado poca rentabilidad, déficit del cereal, desmedidas importaciones y salida de capitales. Esto viene provocando preocupación en los entes gubernamentales y en el sector agrícola-industrial (Zarifian, 1999). El análisis de los factores que inciden en la baja producción y comercialización de los cultivos de maíz de los Agricultores del Cantón Buena Fe, servirá para crear un plan estratégicos y fomentar a los productores a la motivación, fundamentada en su capacidad natural para hacer de la producción una de la mejores en la región, ya que por medio de entrevistas realizadas a los productores de la zona se puede aumentar la confianza de los productores (Giménez \& Muñoz , 2004)

En el Cantón Buena Fe, la población de escasos recursos económicos que realiza una actividad económica agronómica productiva, sobreviven con la venta pequeñas cosechas de granos, frutas, madera, etc. ven relegadas aspiraciones de mejorar su venta de la producción para lograr satisfacer los requerimientos básicos familiares y de sus terrenos (MIPRO, 2014).

Muchos de ellos se han visto obligados a dejar esta actividad y emigrar buscando nuevas oportunidades en otras ciudades del país, por lo que sus hijos han tenido la oportunidad de estudiar pero de igual forma ellos han pasado a formar las masas de ambulantes y en muchos casos de los llamados cinturones de miseria, quedando al margen del rescate de 
su producción y vendiendo sus parcelas en precios muy bajos, con lo que sobreviven en su hogar por un corto tiempo (Corral , 2001).

De ahí, que se hace necesario incentivar la recuperación productiva y búsqueda de formas asociativas que les permitan mantener una calidad de vida digna y solventar las necesidades básicas de su familia. Muchos de estos factores se dan debido a la ignorancia en la gestión de la organización del productor por la presencia de intermediarios con el cual de queda toda producción y en manos los réditos de su gestión (Hernández \& Pulido, 2011). Una agobiante cultura egoísta y de escasa visión de asociación productiva e insuficiente uso de semillas certificadas no permite aprovechar en su totalidad la productividad y comercialización quedando insuficientes recursos para la captación de la producción (Giménez \& Muñoz , 2004).

A estos se suma la desatención del gobierno y el indiscriminado uso de agroquímicos en el sector productivo lo que no permite obtener óptimas condiciones de la producción, provocando desconfianza y desmotivación por sueldos degradados en el campesino que vive de su esfuerzo físico. Se no además la escasa asistencia técnica y políticas que faciliten el abastecimiento del mercado en la región, por lo que se presencia un escaso desarrollo socioeconómico y productivo de la zona (SEMPLADES, 2017).

La falta de políticas gubernamentales claras para el sector agrícola y el poco desarrollo del sector han provocado que los rendimientos de producción del cultivo de maíz a nivel nacional no estén a los niveles de producción de otros países de la región, lo cual implica que seamos un país deficitario de este grano (Giménez \& Muñoz , 2004). En términos generales o en su mayoría, la comercialización de los productos agrícolas se encuentran bajo la acción de los intermediarios, que son los que colocan estos productos en los diferentes mercados, sean éstos locales o internacionales. La fuerte intervención de los intermediarios en la comercialización de estos productos se debe al debilitado sector gremial, lo que hace que esto se vuelva oligopólico y especulativo (Rodriguez \& Bravo, 1991).

El crecimiento de las industrias nacionales de alimentos balanceados en los últimos años y la necesidad de materia prima está obligando a que los agricultores se dedican al cultivo de maíz y consecuentemente busquen mejorar la producción de sus cultivos para tratar de cubrir la demanda de la industria nacional y obtener rentabilidad, pero no se ha logrado ya que existe un alto déficit de esta gramínea, por bajo rendimiento por hectárea, por falta de financiamiento, tecnología, etc. Y la industria se ve en la necesidad de realizar importaciones para cubrir la demanda (Arce , 2009).

\section{Métodos y materiales.}

La investigación se desarrollará en el Nivel Crítico-Propositivo con un diseño de campo, dirigido fundamentalmente al Estudio de los factores que inciden en la baja producción y 
comercialización de los cultivos de maíz de los Agricultores del Cantón Buena Fe, de la Provincia de Los Ríos. En consecuencia el nivel de investigación a realizarse es de tipo Exploratorio, Explicativo y Descriptivo, por cuanto el resultado del presente estudio permitirá conocer como ha incidido la creación de un centro de acopio de raíces y tubérculos, considerando los datos relacionados al tema y la necesidad de mejorar la capacidad de generación de empleo. La muestra de estudio está conformada por 112 productores de maíz del Cantón Buena Fe, de la Provincia de Los Ríos.

La técnica de recolección de datos que se empleara en esta investigación será la encuesta, la cual permitirá recabar la información de los sujetos seleccionados, y las herramientas utilizada para el análisis que se emplearon en la presente investigación Excel, tablas, formulas.

\section{Análisis y discusión de resultados.}

Tabla 1. ¿Existe muchos productores de maíz en el Cantón Buena Fe, de la Provincia de Los Ríos?

\begin{tabular}{ccc}
\hline Opción & Frecuencia & Porcentaje \\
\hline $\mathrm{Si}$ & 101 & 90 \\
$\mathrm{No}$ & 11 & 10 \\
Total & $\mathbf{1 1 2}$ & $\mathbf{1 0 0}$ \\
\hline
\end{tabular}

Elaborado por: Ramiro Guamán.

De acuerdo a las coordinaciones efectuadas, se optó por encuestar a un total de 112 personas involucradas en el ámbito de la presente investigación; de los cuales 101 de ellos manifestaron que SI y mientras que 11 contestaron que no. Los resultados del cuadro anterior indicaron que el $90 \%$ son productores de maíz y el $10 \%$ no son productores de gramíneas. Debe ser porque se dedican a otro tipo de actividad entre ellas a la docencia, enfermería, policías, bomberos y ama de casa.

Tabla 2. ¿Teniendo en cuenta la siguiente escala de hectáreas de cultivos maíz realizadas ¿dónde se ubicaría usted?

\begin{tabular}{lcc}
\hline \multicolumn{1}{c}{ Opción } & Frecuencia & Porcentaje \\
\hline Pequeño (hasta 10 ha.) & 56 & 50 \\
Mediano (de 11 a 50 ha.) & 37 & 33 \\
Grande (+de 50 ha.) & 19 & 17 \\
Total & $\mathbf{1 1 2}$ & $\mathbf{1 0 0}$ \\
\hline
\end{tabular}

Elaborado por: Ramiro Guamán. 
Tabulada la segunda pregunta en relación a teniendo en cuenta la siguiente escala de hectáreas de cultivos maíz realizadas ¿Dónde se ubicaría usted? se obtuvo las siguientes respuestas:

De 112 personas involucradas en el ámbito de la presente investigación; de los cuales 56 de ellos manifestaron que Pequeño (hasta 10 ha.), 37 que Mediano (de 11 a 50 ha.) y mientras que 19 contestaron que Grande (+de 50 ha.). Mediante los resultados de este cuadro, muestra que el $50 \%$ opinaron que son pequeños productores, el $33 \%$ que medianos ya que poseen entre 11 a 50has y un $17 \%$ que grande con más de 50has lo cual le permite apreciar que en la zona predomina la comercialización de la gramínea (maíz) en pequeña escala bajo la dependencia de terceros.

Tabla 3. ¿Cuáles son sus fuentes de financiamiento?

\begin{tabular}{lcc}
\hline \multicolumn{1}{c}{ Opción } & Frecuencia & Porcentaje \\
\hline Bancaria & 11 & 10 \\
Empresa de Agroquímicos & 48 & 43 \\
Capital Propio & 18 & 16 \\
Chulqueros & 35 & 31 \\
\multicolumn{1}{c}{ Total } & $\mathbf{1 1 2}$ & $\mathbf{1 0 0}$ \\
\hline
\end{tabular}

Elaborado por: Ramiro Guamán.

Al observar los resultados arrojados en este cuadro, se nota que el 10\% de los productores utilizan fuentes de financiamiento bancario, $43 \%$ de los productores utilizan fuentes de financiamiento en las empresas de agroquímicos, $16 \%$ de los productores usan sus propias fuentes de financiamiento y $31 \%$ de los productores dependen del chulco. Es notorio que los productores utilizan más el financiamiento mediante empresas de agroquímicos y chulqueros con altos porcentajes de interés, con poco o escaso financiamiento de la banca pública o privada; debe ser por la falta de liquidez, lo que representa falta de garantías para el financiamiento.

Tabla 4. ¿Cómo comercializa su grano?

\begin{tabular}{lcc}
\hline \multicolumn{1}{c}{ Opción } & Frecuencia & Porcentaje \\
\hline $\begin{array}{l}\text { Directamente en las empresas } \\
\text { balanceadoras }\end{array}$ & 28 & 25 \\
Mediante los Intermediarios & 84 & 75 \\
Total & $\mathbf{1 1 2}$ & $\mathbf{1 0 0}$ \\
\hline
\end{tabular}

Elaborado por: Ramiro Guamán. 
El $25 \%$ de los productores les venden sus productos directamente en las empresas balanceadoras y75\% de los productores les venden sus productos mediante los Intermediarios. El mayor porcentaje de los productores de maíz le venden sus productos al mercado mediante los Intermediarios debido a que le ofrece mejores precios y le garantiza la compra de sus productos; mientras que en las balanceadoras deben buscar desde el cupo respectivo hasta el transporte, en conclusión un amplio margen de la ganancia del productor sigue en las manos del intermediario.

Tabla 5. ¿Cuál es su rendimiento Ton/Ha en el cultivo de maíz?

\begin{tabular}{lcc}
\hline \multicolumn{1}{c}{ Opción } & Frecuencia & Porcentaje \\
\hline $2 \mathrm{Ton} / \mathrm{ha}$ & 11 & 10 \\
$3 \mathrm{Ton} / \mathrm{Ha}$ & 22 & 19 \\
$4 \mathrm{Ton} / \mathrm{Ha}$ & 22 & 20 \\
$5 \mathrm{Ton} / \mathrm{Ha}$ & 34 & 30 \\
+6 Ton/ha & 23 & 21 \\
Total & $\mathbf{1 1 2}$ & $\mathbf{1 0 0}$ \\
\hline
\end{tabular}

Elaborado por: Ramiro Guamán.

A un total de 112 personas cabe indicar que 11 de ellos manifestaron que tienen un rendimiento de 2 Ton/ha, 22 que 3 Ton/Ha, 22que 4 Ton/Ha, 34 que 5 Ton/Hay mientras que 23 contestaron que +6 Ton/ha.

Los resultados del cuadro anterior indicaron que el 5\% de los productores manifestaron que tienen un rendimiento de 2 Ton/ha, $19 \%$ que $3 \mathrm{Ton} / \mathrm{Ha}, 20 \%$ que $4 \mathrm{Ton} / \mathrm{Ha}, 30 \%$ que 5 Ton/Hay mientras que $21 \%$ contestaron que +6 Ton/haen rendimiento de sus productos. Esto se debe a que un alto porcentaje agricultores tecnifican sus siembras y cosechas; mientras que el porcentaje restante lo hacen como un cultivo de subsistencia.

Tabla 6. ¿A qué precio comercializa la gramínea?

\begin{tabular}{lcc}
\hline \multicolumn{1}{c}{ Opción } & Frecuencia & Porcentaje \\
\hline Precio Oficial & 28 & 25 \\
Precio de mercado & 84 & 75 \\
Total & $\mathbf{1 1 2}$ & $\mathbf{1 0 0}$ \\
\hline
\end{tabular}

Elaborado por: Ramiro Guamán.

Al observar los resultados arrojados en este cuadro, se nota que el $25 \%$ de los productores vende sus productos a precio oficial, y el $75 \%$ de los productores venden sus productos a precio de mercado La variación de los precios se debe a que a mayor cantidad de venta menor precio, además también varía el precio dependiendo de la calidad del producto y de la responsabilidad del productor en la entrega de la cosecha al intermediario. 
Tabla 7. ¿Cuenta con asistencia técnica y de quién?

\begin{tabular}{lcc}
\hline \multicolumn{1}{c}{ Opción } & Frecuencia & Porcentaje \\
\hline Del gobierno & 22 & 20 \\
Privada & 34 & 30 \\
ONG & 9 & 8 \\
Ninguna & 47 & 42 \\
Total & $\mathbf{1 1 2}$ & $\mathbf{1 0 0}$ \\
\hline
\end{tabular}

Elaborado por: Ramiro Guamán.

El $20 \%$ de los productores cuenta con asistencia técnica del gobierno, 30\% de las empresas privadas de agroquímicos y comercialización el 8\% mediante ONG y42\% de los productores no cuenta con asistencia técnica alguna. Esto se debe a que no tienen la capacitación oportuna para mejorar su productividad y competitividad.

Tabla 8. ¿Cuál de estos factores cree usted que influyen más en la rentabilidad en el cultivo de maíz?

\begin{tabular}{lcc}
\hline \multicolumn{1}{c}{ Opción } & Frecuencia & Porcentaje \\
\hline Crédito oportuno & 28 & 25 \\
Asistencia Técnica & 28 & 25 \\
Insumos de Calidad & 28 & 25 \\
Comercialización de la gramínea & 28 & 25 \\
Total & $\mathbf{1 1 2}$ & $\mathbf{1 0 0}$ \\
\hline
\end{tabular}

Elaborado por: Ramiro Guamán.

De acuerdo a las coordinaciones efectuadas, se optó por encuestar a un total de 112 personas involucradas el 28 manifiestan que crédito oportuno, 28 asistencia Técnica, 28 insumos de Calidad, 28 comercialización de la gramínea. Según los resultados arrojados de este cuadro, se puede observar que los criterios son muy parecidos por lo cual se establece que todos estos factores son imprescindibles en la rentabilidad en el cultivo de maíz.

Tabla 9. ¿Considera que se debe asesorar sobre comercialización al agricultor del cantón Buena Fe?

\begin{tabular}{|c|c|c|}
\hline Opción & Frecuencia & Porcentaje \\
\hline $\mathrm{Si}$ & 86 & 77 \\
\hline No & 26 & 23 \\
\hline Total & 112 & 100 \\
\hline
\end{tabular}

Elaborado por: Ramiro Guamán. 
Según los resultados arrojados de este cuadro, el $77 \%$ de los productores considera que se debe capacitar sobre comercialización al agricultor del cantón Buena $\mathrm{Fe}, 23 \%$ considera que no es necesario capacitar sobre al agricultor. Porque este porcentaje mínimo de productores cosecha muy poca cantidad de la gramínea y no les interesa por que se dedican a otro tipo de actividad, en cambio el mayor porcentaje les interesa y al mismo tiempo es necesario para la comercialización de sus productos.

Tabla 10. ¿Está usted interesado en participar en las actividades que se organicen para el asesoramiento sobre comercialización al agricultor del cantón Buena Fe?

\begin{tabular}{lcc}
\hline \multicolumn{1}{c}{ Opción } & Frecuencia & Porcentaje \\
\hline Si & 81 & $\mathbf{7 2}$ \\
No & 31 & $\mathbf{2 8}$ \\
Total & $\mathbf{1 1 2}$ & $\mathbf{1 0 0}$ \\
\hline
\end{tabular}

Elaborado por: Ramiro Guamán.

Tabulada la décima pregunta en relación a si el agricultor está interesado en participar en las actividades que se organicen para el asesoramiento sobre comercialización al agricultor del cantón Buena Fe se obtuvo las siguientes respuestas:

De acuerdo a las coordinaciones efectuadas, se optó por encuestar a un total de 112 personas involucradas en el ámbito de la presente investigación; de los cuales 81 de ellos manifestaron que si participarían de las actividades para el asesoramiento sobre comercialización al agricultor del cantón Buena Fe, mientras que 31 opinaron que no.

Se puedo observar en los resultados arrojados por este cuadro que el $72 \%$ de los productores estarían dispuesto a participar en las diferentes actividades para el asesoramiento sobre comercialización al agricultor del cantón Buena Fe y el 28\% no tienen mucho interés en participar porque no quieren conocer sobre cómo mejorar la problemática.

Tabla 11. ¿Al ser asesorado sobre comercialización, el agricultor del cantón Buena Fe que beneficio obtendría?

\begin{tabular}{lcc}
\hline \multicolumn{1}{c}{ Opción } & Frecuencia & Porcentaje \\
\hline Obtienen mayor ganancia & 16 & 14 \\
Establece el precio a los diferentes compradores & 16 & 14 \\
Mejora su nivel de vida & 18 & 16 \\
Aumenta las ventas & 53 & 47 \\
Almacena su producto & 9 & 9 \\
Total & $\mathbf{1 1 2}$ & $\mathbf{1 0 0}$ \\
\hline
\end{tabular}

Elaborado por: Ramiro Guamán.

De acuerdo a las coordinaciones efectuadas, se optó por encuestar a un total de 112; los cuales 16 de ellos manifestaron que obtienen mayor ganancia, 16 que se establece el 
precio a los diferentes compradores, 18que mejora su nivel de vida, 53 aseguran que aumenta la venta y mientras que 9 contestaron que solo para almacenar el producto.

El 14\% de los productores obtendría mayor ganancia al ser asesorados, 14\% de los productores establece el precio a los diferentes compradores con la capacitación, $16 \%$ de los productores mejorara su nivel de vida, $47 \%$ de los productores aumentara su venta, 9\% de los productores va almacenar su producto hasta ver cuáles son los resultados del asesoramiento. Todos estos beneficios serían muy importantes para los productores entre ellos el más resaltante es el aumento de las ventas; debe ser porque al ser capacitado sobre comercialización todos venderían sus productos en un solo lugar y los clasificarían y los venderían de acuerdo a su calidad.

\section{Discusión.}

Los puntos fuertes y débiles realizamos una evaluación de la situación de Agricultores y comercializadores de gramínea (maíz) del cantón Buena Fe. Entre los factores externos e internos que de una u otra forma afectan la dinámica socioeconómica de Agricultores y comercializadores de gramínea (maíz) del cantón Buena Fe.

Si cruzamos los factores internos y externos concluyo que se cuenta con potencialidades de grandes, desafíos, riesgos y limitaciones que articulan los diversos componentes de la comercialización.

Tabla 12. Análisis Situacional de los Agricultores del Cantón Buena fe, de la Provincia de Los Ríos.

\begin{tabular}{cc}
\hline \multicolumn{2}{c}{ ANALISIS FODA } \\
\hline \multicolumn{2}{c}{ ANALISIS EXTERNO } \\
OPORTUNIDADES & AMENAZAS \\
Clima & Impuestos \\
Demanda & Políticas de gobierno \\
Déficit & Proveedores de Insumos \\
& Importaciones sin control \\
ANALISIS INTERNO \\
FORTALEZAS & DEBILIDADES \\
Geografía & Comercialización \\
Maquinaria & Asesoramiento \\
Calidad & Financiamiento \\
Ciclo Corto & Almacenamiento \\
\hline
\end{tabular}

Elaborado por: Ramiro Guamán. 


\section{Diagnóstico de la Situación.}

Los principales problemas detectados en este análisis interno y externo FODA son los siguientes:

1. En los factores externos podemos concluir que esta es una zona humedad donde podemos realizar dos siembras de maíz al año y no como en otras que solo se realiza una por zonas secas. Además que existe demanda de la gramínea durante todo el año, y que el país es deficitario de la misma por lo que se debe realizar importaciones, las misma que se realizan en la temporada de poca oferta aunque en años anteriores la realizaban en épocas de cosechas de cosecha con lo que los precios de la gramínea se bajan y los productores perdían la rentabilidad. Podemos decir que no existen políticas claras para el ser debido a los impuestos creados últimamente, aumento en los precios de los agroquímicos, semillas y fertilizantes de manera desmedida, elevando los costos de producción.

2. En los factores internos podemos decir que tenemos una geografía envidiable, pueden mecanizar la mayor parte de los suelos, y ser más eficientes en la producción para obtener grano de calidad en corto tiempo, lo que si existe una escasa asistencia técnica, fuentes de financiamiento para el pequeño y mediano agricultor que caen en la manos de los intermediarios quienes les otorgan crédito a altas tasas de interés, y la comercialización de la gramínea termina en las manos del intermediario que entrega en la industria. La falta de plantas de almacenamiento o de acopio del grano es cuello de botella ya que cuando existe el pico de producción no se puede guardar el excedente, para cuando la oferta de grano baje y poder vender, ya que la demanda de la industria nacional es constante durante todo el año.

\section{Conclusiones.}

- En el cantón Buena Fe, de la Provincia de Los Ríos una gran mayoría de las personas son productoras de maíz, y que existe producción de la gramínea durante invierno y verano, la mayoría son pequeños productores de maíz cual le permite apreciar que en la zona predomina la comercialización de la gramínea (maíz) en pequeña escala bajo la dependencia de terceros. Las fuentes de financiamiento de los productores de maíz son las empresas de agroquímicos y/o los chulqueros con altos porcentajes de interés, con poco o escaso financiamiento de la banca pública o privada; dado por la falta de liquidez, lo que representa falta de garantías para el financiamiento.

- El mayor porcentaje de los productores de maíz le venden sus productos al mercado mediante los Intermediarios debido a que le ofrece mejores precios y le garantiza la compra de sus productos; mientras que en las balanceadoras deben buscar desde el cupo respectivo hasta el transporte, en conclusión un 
amplio margen de la ganancia del productor sigue en las manos del intermediario. Los productores tienen un rendimiento promedio de $5 \mathrm{Ton} / \mathrm{Ha}$ y un alto porcentaje se dedican única y exclusivamente a la siembra y cosecha de estos productos; mientras que el porcentaje restante lo hace en función de ubicar su producto en el mercado.

- La mayoría de los productores no cuenta con asistencia técnica alguna. Esto se debe a que no tienen la capacitación oportuna para mejorar su productividad y competitividad y que factores como crédito oportuno, asistencia Técnica, insumos de Calidad y comercialización de la gramínea son imprescindibles en la rentabilidad en el cultivo de maíz. Es necesario el asesoramiento sobre comercialización al agricultor de cultivo de maíz del cantón Buena Fe, además aseguran que si participarían de las actividades para asesoramiento sobre comercialización de sus productos, de igual forma sus beneficios serían muy importantes, entre ellos el más resaltante es el aumento de las ventas.

\section{Referencias.}

Hada , D. (15 de abril de 2008). La Nueva Dinámica del Mercado de los Commodities. Obtenido de Banco Central de Reserva.: http://www.bcr.gob.sv/bcrsite/uploaded/content/category/611872653.pdf

Arce, S. (12 de Noviembre de 2009). Identificacion de los Principales Problemas en la Logistica de Abastecimiento de las Empresas Constructoras Bogotanas y Propuesta de MejoraS. tesis de Pregrado PONTIFICIA UNIVERSIDAD JAVERIANA, 173. Bogota, Colombia. Obtenido de http://javeriana.edu.co/biblos/tesis/economia/tesis189.pdf

CEPAL. (2016). Perspectivas de la agricultura y del desarrollo rural en las Américas. San José: IICA, Sede Central. Obtenido de http://www.iica.int/sites/default/files/publications/files/2015/b3695e.pdf

Corral , A. (4 de agosto de 2001). Manual de Auditoria Financiera Gubernamental. Obtenido de contraloria.gob.ec: http://www.contraloria.gob.ec/documentos/normatividad/ManAudFin.pdf

Giménez , G., \& Muñoz , V. (2004). CORTINA, Adela: "Hasta un Pueblo de Demonios"Ética Pública y Sociedad. Ed.Taurus. Pensamiento. Revista Confluencia, $1(4), \quad 10 . \quad$ Obtenido de http://bdigital.uncu.edu.ar/objetos_digitales/333/Gimenez.ResenaConfluencia4.p df

Hernández , S., \& Pulido, A. (2011). Fundamentos de gestión empresarial. México D.F.: McGRAW-HILL/INTERAMERICANA. Obtenido de 
https://www.academia.edu/16578340/272437730-Fundamentos-de-Gestion-

Empresarial

MIPRO. (4 de diciembre de 2014). Proyecto Nacional para el Desarrollo Integral de Cadenas Agroindustriales. Obtenido de Miniterio de industrias y productividad: http://www.industrias.gob.ec/wp-content/uploads/2015/09/PROYECTONACIONAL-PARA-EL-DESARROLLO-INTEGRAL-DE-CADENASAGROINDUSTRIALES.pdf

Philip , K., \& Gary , A. (2012). Marketing (Decima cuarta ed.). México: Pearson Educación.

https://profdariomarketing.files.wordpress.com/2014/03/marketing_kotlerarmstrong.pdf

Rodriguez , F., \& Bravo, L. (1991). Indicadores de productividad en la empresa. Venezuela: Nuevos Tiempos. Obtenido de http://scioteca.caf.com/bitstream/handle/123456789/863/Indicadores\%20de\%20 calidad\%20y\%20productividad\%20en\%20la\%20empresa.PDF

Sanchez, R. (Granada de 2013). Implementación de la gestión por competencias para el desarrollo de la gestión del conocimiento en servientrega S.A. Tesis de pregrado UNIVERSIDAD NACIONAL ABIERTA Y A DISTANCIA “UNAD”, 58. Obtenido de

http://stadium.unad.edu.co/preview/UNAD.php?url=/bitstream/10596/1251/1/40 449089.pdf

SEMPLADES. (2017). Secretaria Nacional del Planificacion y desarrollo. Obtenido de Semplades: http://www.planificacion.gob.ec/wpcontent/uploads/downloads/2017/10/PNBV-26-OCT-

FINAL_0K.compressed1.pdf

Zarifian, P. (1999). Mutación de los sistemas productivos y competencias profesionales. montevideo: Cinterfor. Obtenido de oitcinterfor.org: https://www.oitcinterfor.org/sites/default/files/file_publicacion/papel8.pdf

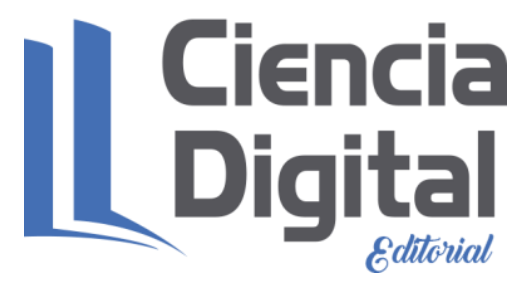




\section{Para citar el artículo indexado.}

Guamán R., Alcívar E., López M. \& Quiñónez L. (2018). Estudio de los factores que inciden en la baja producción y comercialización de los cultivos de maíz. Revista electrónica Visionario Digital1(1), 50-64. Recuperado desde: http://cienciadigital.org/revistacienciadigital2/index.php/VisionarioDigital/article/view/36/ $\underline{35}$

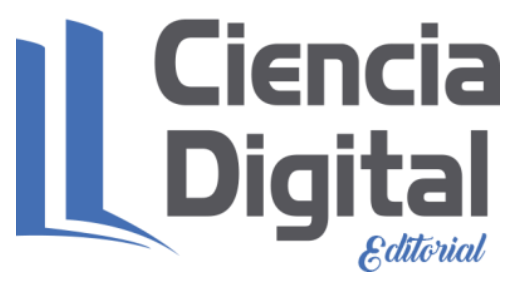

El artículo que se publica es de exclusiva responsabilidad de los autores y no necesariamente reflejan el pensamiento de la Revista Ciencia Digital.

El articulo queda en propiedad de la revista y, por tanto, su publicación parcial y/o total en otro medio tiene que ser autorizado por el director de la Revista Ciencia
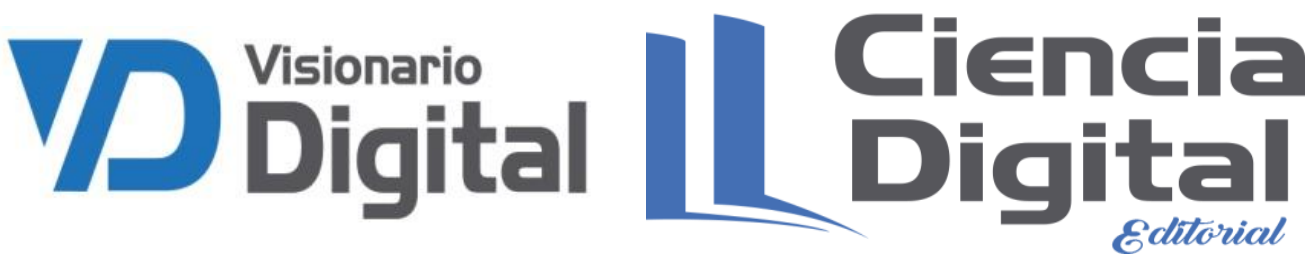